\title{
Development of Ligase-Assisted Spacer Addition for the Measurement of Microsatellites
}

BioTechniques 31:__-_(July 2001)

\author{
V. Brockhurst, R. Barnard ${ }^{1}$, \\ L. Wolter, P. Giffard, and \\ P. Timms \\ CRC for Diagnostic Technolo- \\ gies, Queensland University of \\ Technology, Brisbane, and \\ ${ }^{1}$ University of Queensland, \\ St. Lucia, Australia
}

\section{INTRODUCTION}

Microsatellites, otherwise known as simple sequence repeats (SSRs), short tandem repeats (STRs), or simple sequence-length polymorphisms (SSLPs) consist of repetitive tracts of short DNA core sequences, of which the core units are generally between 1 and $6 \mathrm{bp}$. They are ubiquitous in eukaryotic genomes and are highly polymorphic because of variation in the number of repeat units they contain (20). It is this hypervariability that has prompted their widespread use as DNA markers in forensics $(3,6)$, gene mapping $(4,8)$, population studies addressing a wide range of questions including individual identification (11), relatedness, parentage $(1,12,14)$, and intraspecies comparisons (16), and medical diagnostics, dealing with a new class of neurodegenerative diseases associated with trinucleotide instability $(2,5,15,18)$.

One commonly used method for detecting length variability consists of resolving PCR-amplified alleles on $6 \%$ denaturing polyacrylamide sequencing gels and visualization by autoradiography, either by PCR primers end-labeled with radionucleotides or radioactively labeled deoxynucleotides incorporated during amplification. Several modifications have aimed to simplify this procedure, including alternative staining methods $(7,17,22,24)$, modified gel matrices to increase the level of resolution (9), and the recycling of polyacrylamide gel electrophoresis (PAGE) gels to reduce the cost of reagents and time (23).

One major problem with using denaturing gel electrophoresis is that it is a time-consuming and labor-intensive technique and, thus, is not suitable for large throughput of samples. Multiplexing of several microsatellite loci has been able to reduce the time required but often requires considerable optimization (10). Several techniques have recently emerged to circumvent these problems. Fluorescent gel scanning utilizing fluorescent dyes attached to PCR primers has provided an alternative detection system and has lent itself to automation, although it is still dependent on the use of polyacrylamide gels and dedicated instrumentation (Model 672 Genescanner System; Applied Biosystems, Foster City, CA, USA) (21). Capillary electrophoresis allows for a more rapid separation of DNA fragments when analyzing a small number of samples and provides resolution of units differing by as little as $1 \mathrm{bp}$ (13), comparable to denaturing gel electrophoresis. Time of flight mass spectroscopy (TOF-MS) has long been used to measure molecular weights. Attempts to resolve microsatellite alleles in this manner have been promising; however, resolution remains poor at sizes greater than $60 \mathrm{bp}$ (19).

There is thus a critical need for the development of new, rapid, and reliable techniques for analyzing length polymorphisms, ultimately leading to suitability for routine laboratory use. In this paper, we describe such a novel method, utilizing an ELISA-based format.

\section{MATERIALS AND METHODS}

\section{Source of Tetranucleotide- Containing Microsatellites for Method Development}

The MM211 microsatellite is found within the genome of the Major 
Mitchell Cockatoo (Cacatua leadbeateri) and contains the tetrameric sequence ATCC (PCR conditions were provided by David Groth from the University of Western Australia). PCR conditions for amplifying the 180-bp fragment were as follows: $1.5 \mathrm{mM}$ $\mathrm{MgCl}_{2}, 200 \mu \mathrm{M}$ dNTP, 100 ng genomic DNA, $0.4 \mathrm{mM}$ each primer MM211F (5'-AGATAATCCTTGAGGTCCCTT$\left.3^{\prime}\right)$ and MM211R (5'-GCCCAAAGTCTGCCTCCCATTC-3'), 0.5 U Taq DNA polymerase (Applied Biosystems). Cycling parameters consisted of an initial denaturation at $94^{\circ} \mathrm{C}$ for 5 min, 35 cycles of $94^{\circ} \mathrm{C}$ for $30 \mathrm{~s}, 55^{\circ} \mathrm{C}$ for $30 \mathrm{~s}$, and $72^{\circ} \mathrm{C}$ for $30 \mathrm{~s}$, with a final extension at $72^{\circ} \mathrm{C}$ for $7 \mathrm{~min}$.

PCR product was generated from Cockatoo sample 48404, gel-purified using GENECLEAN ${ }^{\circledR}$ (Bio 101, Vista, CA, USA) and cloned into the pGEM $^{\circledR}$-T vector (Promega, Madison, WI, USA). The insert was sequenced and contained a region with 10 repeats of ATCC. This clone was used in all optimization experiments.

Specimens Containing 9, 10, 11, or 12 Tetranucleotide Repeats

Eleven Major Mitchell Cockatoo genomic DNA samples were initially genotyped using the traditional $6 \%$ denaturing polyacrylamide gel electrophoresis method and detected via autoradiography. Samples were shown to contain both homozygous and heterozygous genotypes containing either 9, 10,11 , or 12 ATCC repeats.

\section{Ligase-Assisted Spacer Addition Methodology}

General method description. The ligase-assisted spacer addition (LASA) method relies on the use of three oligonucleotides, two of which flank the repeat region (oligonucleotide "us" and oligonucleotide "ds"). Oligonucleotide "us" has a moiety at its 5 ' end to allow attachment to a solid support (such as biotin for capturing onto streptavidin-coated microtiter wells). Oligonucleotide "ds" has a chromophore attached at its 3 ' terminus (such as fluorescein) for detection purposes. A selection spacer oligonucleotide is also used, being complementary to the tandem repeat core unit. Varying lengths of this spacer oligonucleotide are added in separate reactions (e.g., well 1 uses a 9-repeat, well 2 uses a 10repeat, etc.), such that one will be of the same length as that of the repeat region being analyzed.

The method involves the initial denaturation of the template (PCR product spanning the microsatellite region) and subsequent hybridization of the three oligonucleotides. A ligation reaction follows resulting in the joining of oligonucleotide "us" to the correct spacer oligonucleotide and to the oligonucleotide "ds". The ligation product is captured onto a solid support, and the original template is removed with alkali treatment. Successful ligation of all three oligonucleotides will result in the detection of fluorescence and will correspond to one spacer oligonucleotide length, thereby indicating the length of the repeat region (Figure 1). A final, optimized protocol incorporating all of the optimized parameters undertaken in this study is detailed in Table 1.

\section{Development and Optimization of the LASA Method}

Many sequential modifications were made to the methodology to more fully characterize the reaction kinetics and to optimize its performance. We describe herein only several important factors influencing the success of this technique.

\section{Preliminary LASA Conditions}

Noncycling conditions consisted of one denaturation step at $94^{\circ} \mathrm{C}$ for $5 \mathrm{~min}$, with a ligation step at $65^{\circ} \mathrm{C}$ for $60 \mathrm{~min}$, with reaction products initially being bound and detected on streptravidincoated dynabeads (Dynal, Lake Success, NY, USA). To facilitate assay handling, LASA reaction products were instead transferred onto streptavidincoated microplates (NEN Life Science Products, Boston, MA, USA). To achieve high detection levels, cycling of the two-step denaturation/ligation reactions was conducted, involving an initial denaturation at $94^{\circ} \mathrm{C}$ for 5 min with 99 rounds of a two-step temperature cycle

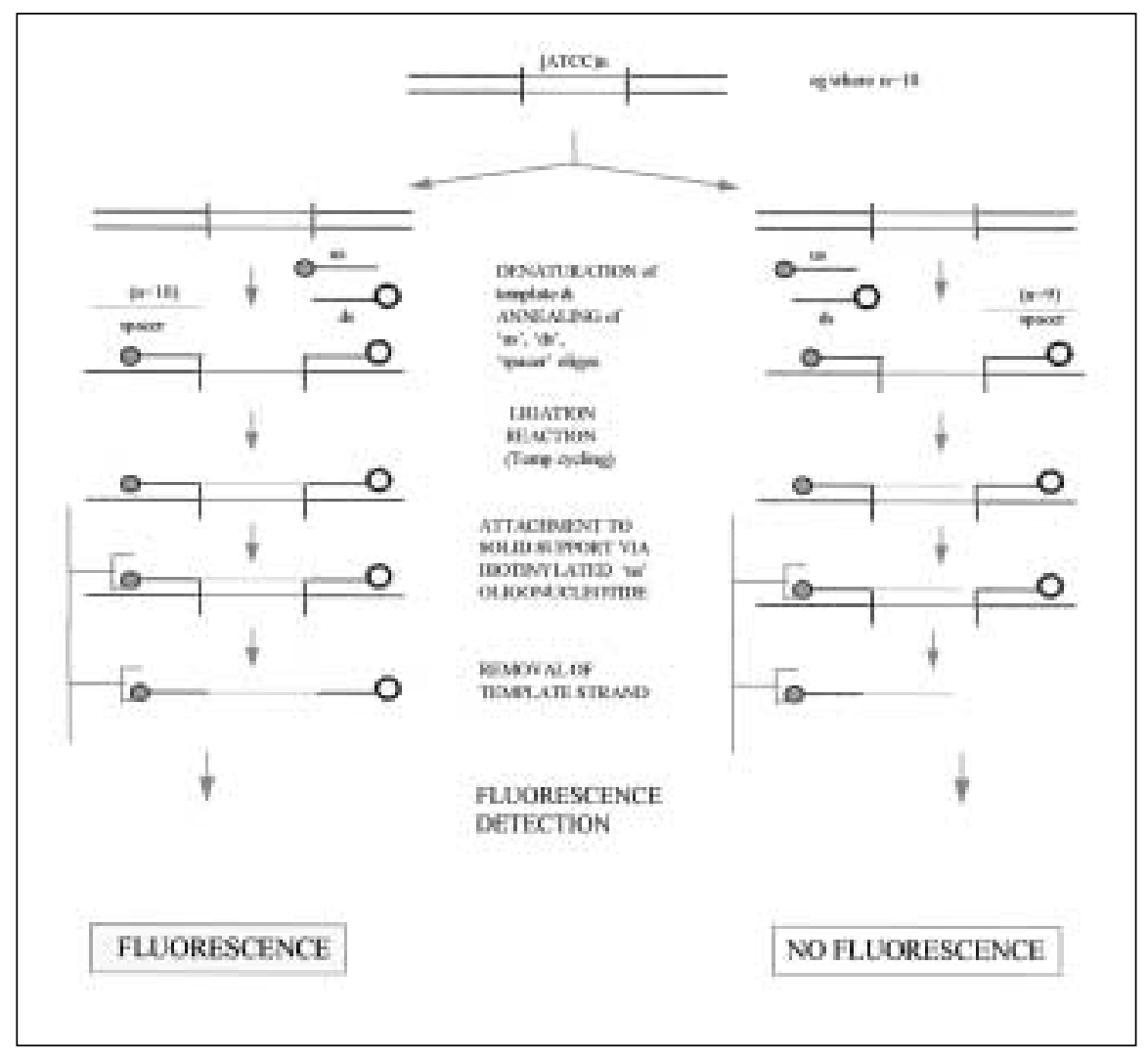

Figure 1. Schematic representation of the LASA methodology. 


\section{Research Report}

Table 1. LASA Protocol

1. In a reaction vial, $20 \mathrm{pmol}$ of the oligonucleotide "us", oligonucleotide "ds", and "spacer" oligonucleotides, and 0.2 pmol unbiotinylated competitive oligonucleotides are added with $1 \mu \mathrm{L} \mathrm{PCR}$ product and $4 \mathrm{U}$ Ampligase (thermostable ligase; Epicentre Technologies, Madison, WI, USA) and appropriate buffer in a final reaction volume of $20 \mu \mathrm{L}$.

2. The reaction mixture is initially denatured at $94^{\circ} \mathrm{C}$ for $5 \mathrm{~min}$, with a subsequent 25 cycles of ligation and denaturation steps (i.e., $65^{\circ} \mathrm{C}$ for $60 \mathrm{~s}$ and $94^{\circ} \mathrm{C}$ for $10 \mathrm{~s}$ for 25 cycles).

3. Sixty microliters of a stop buffer ( $20 \times$ SSC containing $30 \mathrm{mM}$ EDTA) are added, and each reaction mixture is added to a streptavidin-coated microtiter well. Binding is allowed to proceed for $30 \mathrm{~min}$ at room temperature.

4. Two 0.1 M NaOH washes are followed by six washes in PBS/0.1\% Tween 20.

5. An antifluorescein-alkaline phosphatase conjugate is diluted 1:1000 in 1\% skim milk in $100 \mathrm{mM} \mathrm{Tris,} \mathrm{pH} 7.5 / 150 \mathrm{mM} \mathrm{NaCl}$. After a 30-min incubation at room temperature, the wells are washed six times with PBS $/ 0.1 \%$ Tween 20 and three times with PBS.

6. One hundred microliters of p-nitrophenyl phosphate (PnPP) substrate (Sigma, St. Louis, MO, USA) is added, and color is left to develop. An absorbance reading at $405 \mathrm{~nm}$ at $15-120 \mathrm{~min}$ is taken.

Table 2. LASA Oligonucleotides Designed for the MM211 Microsatellite Detection

\begin{tabular}{|ll|}
\hline Oligonucleotides & Sequence \\
\hline ATCC-us & 5'-Biotinylated -GATTCTGTGATTCTACAACC-3' \\
ATCC-ds & 5'-Phosphorylated-ACCCACAGACCTCTTCCCAC-3' Fluorescein \\
ATCC-4 & 5'-Phosphorylated-ATCCATCCATCCATCC-3' \\
ATCC-9 & 5'-Phosphorylated-ATCCATCCATCCATCCATCCATCCATCCATCCATCC-3' \\
ATCC-10 & 5'-Phosphorylated-ATCCATCCATCCATCCATCCATCCATCCATCCATCCATCC-3' \\
ATCC-11 & 5'-Phosphorylated-ATCCATCCATCCATCCATCCATCCATCCATCCATCCATCCATCC-3' \\
ATCC-12 & 5'-Phosphorylated- ATCCATCCATCCATCCATCCATCCATCCATCCATCCATCCATCCATCC-3' \\
Competitive-us+9 & 5'-GATTCTGTGATTCTACAACCATCCATCCATCCATCCATCCATCCATCCATCCATCC-3' \\
Competitive-us+10 & 5'-GATTCTGTGATTCTACAACCATCCATCCATCCATCCATCCATCCATCCATCCATCC-3' \\
Competitive-us+11 & 5'-GATTCTGTGATTCTACAACCATCCATCCATCCATCCATCCATCCATCCATCCATCCATCCATCC-3' \\
Competitive-us+12 & 5'-GATTCTGTGATTCTACAACCATCCATCCATCCATCCATCCATCCATCCATCCATCCATCCATCCATCC-3' \\
\hline
\end{tabular}

of $65^{\circ} \mathrm{C}$ for $1 \mathrm{~min}$ and $94^{\circ} \mathrm{C}$ for $10 \mathrm{~s}$.

Ligation temperature. Replicate LASA reactions were run on a Temperature Gradient PCR instrument (MJ Research, Waltham, MA, USA) to ascertain the optimal ligation temperature $\left(41^{\circ} \mathrm{C}-79^{\circ} \mathrm{C}\right)$.

Varying amount of denaturation/ ligation cycling. Replicate LASA reactions were performed using 20 pmol of all three oligonucleotides and using the conditions described above, with 1, 5, $10,25,50$, and 99 rounds of denaturation/ligation cycles.

Optimization of template concentration. Varying quantities of PCR product $(25-0.08 \mu \mathrm{L})$ were used in LASA reactions to determine the working range of template concentration for this assay. For all LASA optimizations carried out previously, $5 \mu \mathrm{L}$ PCR product were used.

\section{Southern Blot Analysis for Confirmation of the Sizes of Spacer Oligonucleotides Incorporated during the LASA Reaction}

Southern blotting of sequencing gels. Ten microliters of each LASA reaction (ATCC-9, ATCC-10, ATCC-11, and ATCC-12) was loaded onto a $6 \%$ denaturing polyacrylamide gel and electrophoresed at $50 \mathrm{~W}$ at $60^{\circ} \mathrm{C}$ for $1.5 \mathrm{~h}$ on a sequencing gel apparatus (Life Technologies, Rockville, MD, USA) before Southern blotting onto a Hybond ${ }^{\circledR} \mathrm{N}^{+}$ membrane (Amersham Pharmacia Biotech, Piscataway, NJ, USA) overnight using running buffer (TBE). The blot was briefly rinsed in $2 \times$ standard saline citrate (SSC) and blocked for $30 \mathrm{~min}$ in $1 \%$ blocking reagent (Roche Molecular Biochemicals, Indianapolis, IN, USA) in buffer 1 ( $0.1 \mathrm{M}$ maleic acid, $0.15 \mathrm{M}$ $\mathrm{NaCl}, \mathrm{pH}$ 7.5). The blot was subsequently incubated for $30 \mathrm{~min}$ in a streptavidin-alkaline phosphatase conjugate (Roche Molecular Biochemicals) diluted 1:20000 in 1\% blocking reagent to detect biotinylated products. Following two 15 -min washes in $0.3 \%$ Tween ${ }^{\circledR} 20$ in buffer 1 , the blot was equilibrated in buffer 3 (0.1 M Tris, pH 9.5, 0.1 M $\mathrm{NaCl}$ ), and the bands were visualized by adding diluted CDP-Star ${ }^{\mathrm{TM}}$ (Roche Molecular Biochemicals) (1:100 in buffer 3 ) and X-ray exposure.

Titration of non-biotinylated competitive oligonucleotides in a compet- 
Table 3. Exemplary Initial LASA Result without Any Optimizations

\begin{tabular}{|c|c|c|c|}
\hline SPACER & Replicate No. 1 & Replicate No. 2 & Replicate No. 3 \\
\hline No spacer & 0.038 & 0.043 & 0.040 \\
\hline ATCC-9 & 1.553 & 1.720 & 1.939 \\
\hline ATCC-10 & 3.779 & 3.458 & 3.510 \\
\hline ATCC-11 & 0.922 & 1.305 & 0.825 \\
\hline ATCC-12 & 0.987 & 0.818 & 0.969 \\
\hline \multicolumn{4}{|c|}{$\begin{array}{l}\text { The target template used was a PCR product generated from a clone known to } \\
\text { contain } 10 \text { repeats of the tetramer ATCC. Four separate reactions containing one } \\
\text { of four selective spacer oligonucleotides (ATCC-9, ATCC-10, ATCC-11, and } \\
\text { ATCC-12) and two common upstream and downstream oligonucleotides are un- } \\
\text { dertaken. Cycling at two temperatures encourages the denaturation of the tem- } \\
\text { plate strands and the hybridization/ligation of the upstream and downstream } \\
\text { oligonucleotides to the spacer oligonucleotide matching the length of the mi- } \\
\text { crosatellite repeat region. Capture via a biotinylated moiety on the upstream } \\
\text { oligonucleotide and detection via a fluorophore attached to the downstream } \\
\text { oligonucleotide provide evidence for the ligation event. Three replicates show } \\
\text { high absorbance signals for the correctly matched spacer oligonucleotide (shad- } \\
\text { ing corresponds to the correct spacer length). Background signals for the mis- } \\
\text { matched spacer molecule are, however, still high and therefore unacceptable. }\end{array}$} \\
\hline
\end{tabular}

itive LASA format. For each of the LASA reactions (ATCC-9, ATCC-10, ATCC-11, and ATCC-12), nonbiotinylated competitive oligonucleotides were included. The sequences of the competitive oligonucleotides were derived by combining the sequences of the upstream oligonucleotide (oligonucleotide "us") and of a spacer oligonucleotide of length other than the biotinylated selection spacer oligonucleotide. Thus in the "9" reaction well, a biotinylated ATCC-9 spacer oligonucleotide was added with three nonbiotinylated competitive oligonucleotides; that is, "us" $+(\text { ATCC })_{10}$, "us"+(ATCC) $)_{11}$, and "us"+(ATCC $)_{12}$ oligonucleotides (Table 2). A titration of equimolar amounts of each competitive oligonucleotide was investigated to ascertain the optimal concentration to reduce background signals.

\section{RESULTS}

\section{Optimization of the Critical LASA Parameters}

In all of the optimizations, the target template used was a PCR product generated from a clone known to contain 10 repeats of the tetramer ATCC. The PCR product was thus expected to mainly contain 10 tetrameric repeats, with the possibility of other minor products due to replication slippage (stutter bands) containing greater or less than the (ATCC) 10 repeats.

Preliminary LASA result (without optimizations). Incorporation of the correctly matched spacer oligonucleotide showed high absorbance levels, discriminating against that of the incorrect spacer oligonucleotides in all triplicates (Table 3). However, the incorporation signals from the mismatched spacer oligonucleotides were moderately high, ranging between $23.6 \%$ and $55.2 \%$ of the correctly incorporated spacer oligonucleotide. Note that "background" refers to the signal generated by mismatched spacer oligonucleotides. Various parameters were investigated to achieve optimal assay conditions and highly discriminatory signals. These are described henceforth.

Ligation temperature. The highest absorbance readings were observed over the range $41^{\circ} \mathrm{C}-56.8^{\circ} \mathrm{C}$, with a gradual decrease in readings from $56.8^{\circ} \mathrm{C}$ to $72.5^{\circ} \mathrm{C}$ (Table 4 ). At $72.5^{\circ} \mathrm{C}$, no reaction products were evident, even following an overnight color development. However, it was noted that across the temperature range, misincorporation background readings for the other three spacer inserts were also elevated, 


\section{Research Report}

with a gradual decline in their intensities with increasing temperature. The highest ratio of correct signal to false mize signal-to-noise ratios, all LASA reactions were subsequently performed using a ligation temperature of $65^{\circ} \mathrm{C}$.

Varying the amount of denaturation/ligation cycling. A nonlinear amplification profile was observed with increasing cycle number (data not shown). At 10 cycles, absorbance signals were amplified approximately 9fold $(0.145-0.923)$ relative to one cycle. However, further increases did not result in a proportional rise in absorbance reading. A further 2-fold signal amplification was achieved by altering the cycle number from 10 to 150 . Twenty-five cycles were thus deemed sufficient for this assay.

Template concentration. Comparable signals were observed with PCR signal was observed at $65^{\circ} \mathrm{C}$. To maxi-

Table 4. Temperature Titration for the Ligation Step

\begin{tabular}{|c|c|c|c|c|c|c|c|}
\hline \multirow[b]{2}{*}{ Spacer } & \multicolumn{7}{|c|}{ Absorbance $405 \mathrm{~nm}$} \\
\hline & $55.6^{\circ} \mathrm{C}$ & $56.8^{\circ} \mathrm{C}$ & $58.8^{\circ} \mathrm{C}$ & $61.6^{\circ} \mathrm{C}$ & $65.0^{\circ} \mathrm{C}$ & $69.0^{\circ} \mathrm{C}$ & $72.5^{\circ} \mathrm{C}$ \\
\hline ATCC-9 & 1.638 & 1.297 & 1.968 & 1.001 & 0.320 & 0.072 & 0.049 \\
\hline ATCC-10 & 2.984 & 3.333 & 2.488 & 2.212 & 1.078 & 0.525 & 0.047 \\
\hline ATCC-11 & 2.061 & 1.548 & 0.832 & 0.274 & 0.072 & 0.047 & 0.045 \\
\hline ATCC-12 & 0.281 & 0.481 & 0.131 & 0.119 & 0.053 & 0.051 & 0.042 \\
\hline \multicolumn{8}{|c|}{$\begin{array}{l}\text { Temperature titration for the ligation step }\left(55.6^{\circ} \mathrm{C}-72.5^{\circ} \mathrm{C}\right) \text { shows a decrease in } \\
\text { signals for the correctly matched spacer oligonucleotide. However, background } \\
\text { absorbance readings are reduced to a greater extent with increasing tempera- } \\
\text { ture, indicating a temperature-related dependence for the addition of the mis- } \\
\text { matched spacer oligonucleotide. }\end{array}$} \\
\hline
\end{tabular}

product volumes as low as $0.05 \mu \mathrm{L}$ PCR product (equivalent to approximately 250 fmol product). In addition, background signals decreased accordingly (Table 5).

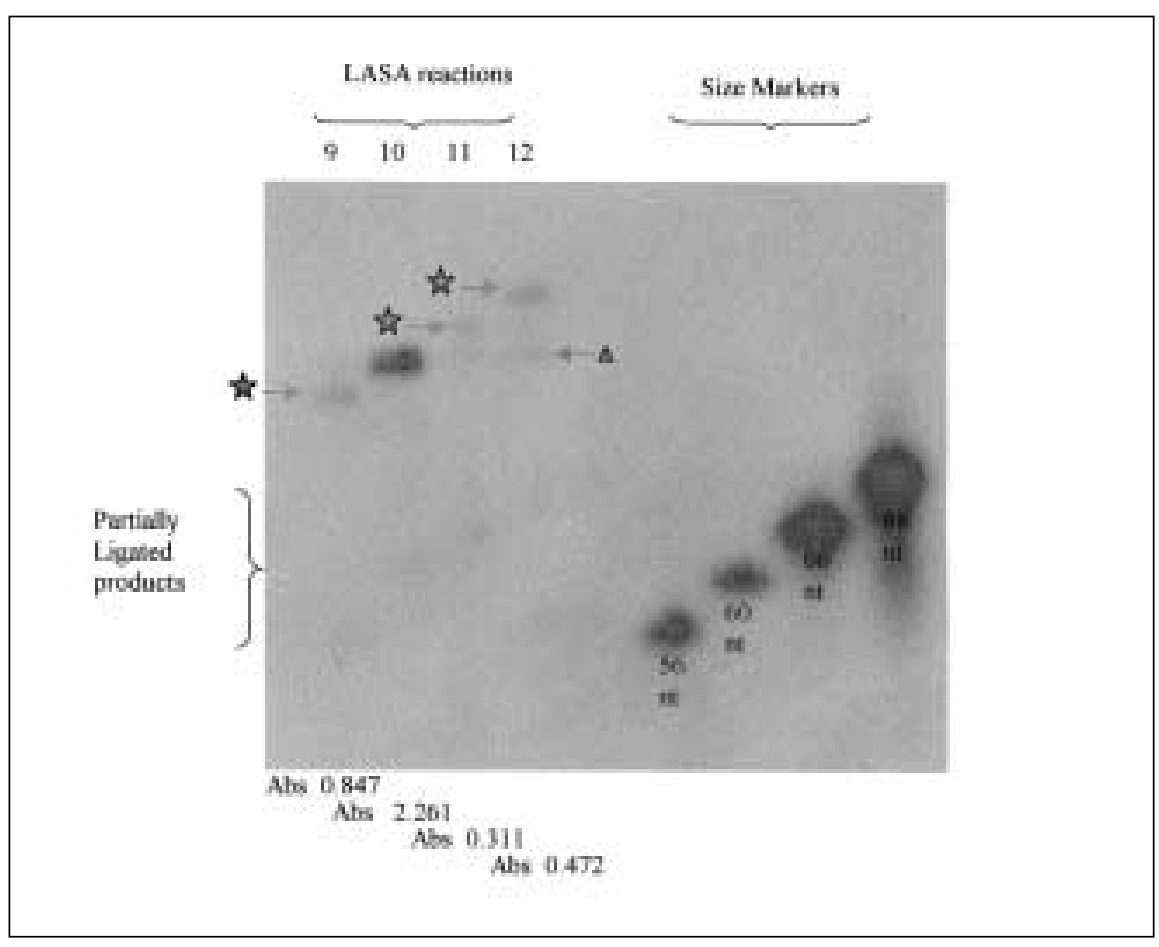

Figure 2. Southern blot of a $6 \%$ denaturing PAGE gel of each LASA reaction probed with a streptavidin-alkaline phosphatase conjugate to detect biotinylated products. An identical blot was also probed with an antifluorescein-alkaline phosphatase conjugate and revealed an identical profile (data not shown). Products in each reaction were comprised of partially ligated products (upstream and spacer oligonucleotides) and fully ligated products as shown by the stars. Sizing of full products are consistent with the ligation of the respective spacer oligonucleotide to the two flanking common oligonucleotides in each of the reactions. It is thus a strong possibility that the template strand has modified its structure to narrow the proximity of the spacer oligonucleotide and the common flanking oligonucleotides to permit ligation. We hypothesize that stem-loop formation would allow such an occurrence, particularly due to the repetitive nature of the sequence. An additional band (as shown by a triangle) is visible in three of the reactions (ATCC-9, ATCC-11, and ATCC-12), being equivalent to a length of ATCC-10. The possibility of contamination is to be considered.

\section{Elimination of False LASA Incorporation Signals}

Although these various modifications significantly improved the level of incorporation of the correct spacer insert (ATCC-10), we observed a consistently elevated background level with the other spacer inserts. In particular, of the three incorrect spacer incorporations, background readings for the (ATCC-9) spacer oligonucleotide were the highest. Furthermore, when cloned DNA (not a PCR product) containing the 10 tetrameric repetitive sequence was used in the LASA reaction, background signals were still evident (data not shown). This suggested that the presence of PCR stutter bands was not solely responsible for the background readings observed when using PCR product in the LASA assay. We subsequently evaluated whether secondary structures, such as stem-loop formation, might be contributing to this false incorporation.

\section{Evidence Substantiating False Incorporation Due to Stem-Loop Formation}

LASA products (from both PCR products and cloned DNA) were electrophoresed on a long $6 \%$ denaturing PAGE gel, Southern blotted, and probed for the fully ligated product. A strong signal was evident in the "10" reaction, equivalent to a fully ligated LASA product containing 10 ATCC repeats (Abs of 2.261) (Figure 2). The other three reactions (ATCC-9, -11, and -12) showed bands of lower intensities (comparable 


\section{Research Report}

Table 5. Titration of the Template Concentration in the LASA Assay

\begin{tabular}{|lcccccccccccc}
\hline & \multicolumn{10}{c|}{ Absorbance $\mathbf{4 0 5} \mathbf{~ n m}$} \\
\cline { 2 - 12 } & $\mathbf{0}$ & $\begin{array}{c}\mathbf{5} \\
\mathbf{f m o l}\end{array}$ & $\begin{array}{c}\mathbf{5 0} \\
\mathbf{f m o l}\end{array}$ & $\begin{array}{c}\mathbf{1 0 0} \\
\mathbf{f m o l}\end{array}$ & $\begin{array}{c}\mathbf{2 5 0} \\
\mathbf{f m o l}\end{array}$ & $\begin{array}{c}\mathbf{5 0 0} \\
\mathbf{f m o l}\end{array}$ & $\begin{array}{c}\mathbf{1} \\
\mathbf{p m o l}\end{array}$ & $\begin{array}{c}\mathbf{5} \\
\mathbf{p m o l}\end{array}$ & $\begin{array}{c}\mathbf{1 0} \\
\mathbf{p m o l}\end{array}$ & $\begin{array}{c}\mathbf{2 5} \\
\mathbf{p m o l}\end{array}$ & $\begin{array}{c}\mathbf{5 0} \\
\mathbf{p m o l}\end{array}$ & $\begin{array}{c}\mathbf{1 0 0} \\
\mathbf{p m o l}\end{array}$ \\
\hline ATCC-9 & 0.071 & 0.073 & 0.103 & 0.176 & 0.501 & 0.727 & 0.891 & 1.241 & 1.103 & $\mathbf{1 . 2 9 8}$ & 0.165 & 0.132 \\
ATCC-10 & 0.114 & 0.251 & 0.479 & 0.710 & 1.772 & 2.005 & 2.268 & 2.910 & 1.318 & $\mathbf{2 . 4 4 5}$ & 2.697 & 0.627 \\
ATCC-11 & 0.041 & 0.044 & 0.057 & 0.057 & 0.072 & 0.247 & 0.189 & 0.166 & 0.265 & $\mathbf{0 . 1 0 7}$ & 0.094 & 0.191 \\
ATCC-12 & 0.046 & 0.048 & 0.058 & 0.058 & 0.082 & 0.285 & 0.138 & 0.231 & 0.098 & $\mathbf{0 . 1 9 6}$ & 0.051 & 0.144
\end{tabular}

Titration of the template concentration in the LASA assay also demonstrates a relationship between the amount of template and the level of background noise, with a larger background signal observed in the spacer oligonucleotide, which is smaller relative to the correct spacer oligonucleotide. A typical $5-\mu \mathrm{L}$ volume of PCR product generates a signal-to-noise ratio of approximately 1:2 (ATCC-9:ATCC-10). A 1:100 dilution of the template still provides high detection levels, however, with reduced background.

Bold font indicates $5 \mu \mathrm{L}$ PCR product used in the reaction.

Table 6. Competitive Oligonucleotide Concentration

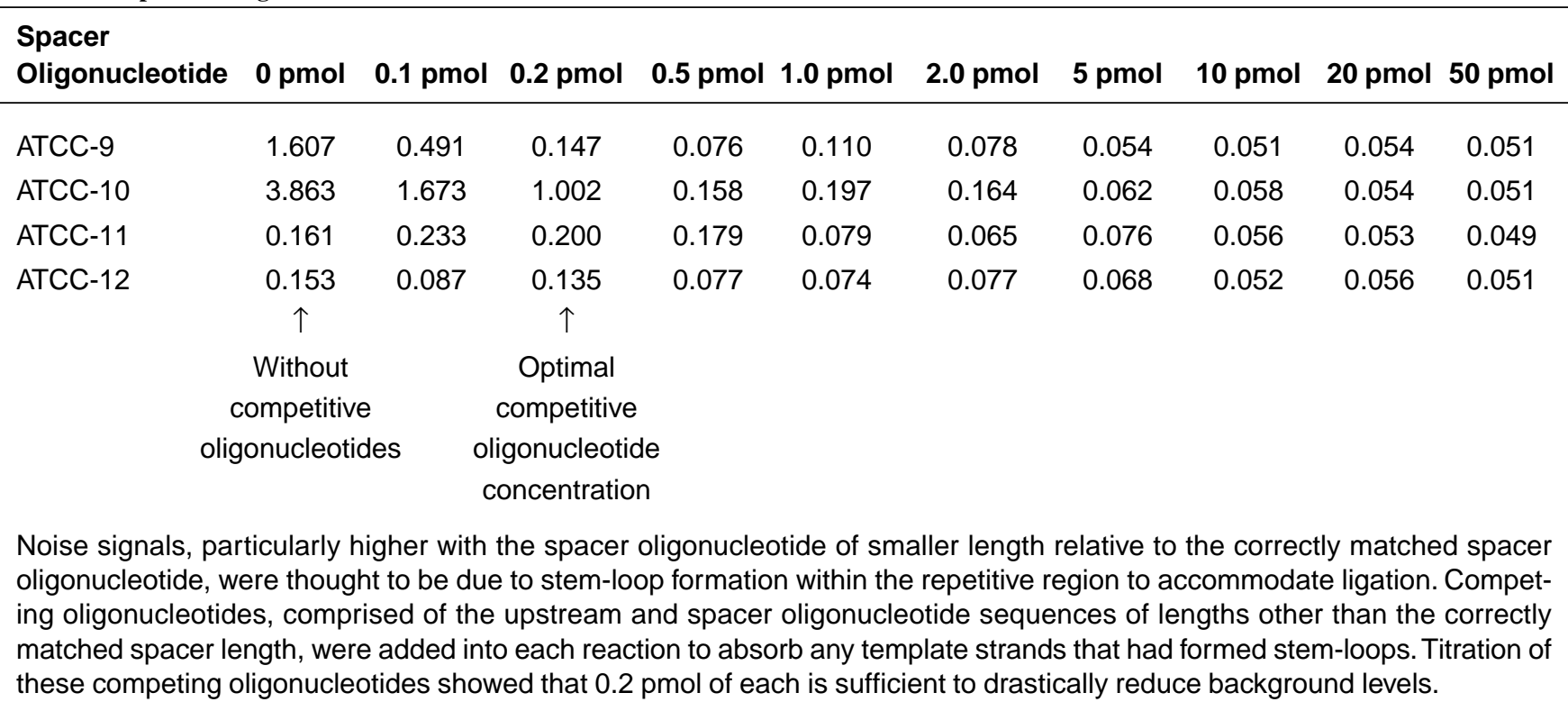

to their absorbance readings) and equivalent to lengths of 9,11 , or 12 repeats (as shown by * arrows). These same products, with similar relative intensities, were also observed when cloned DNA was used as the template for a LASA reaction (data not shown). This result suggests the formation of hairpin loops within the repetitive region was responsible for the incorporation of the incorrect spacer oligonucleotides.

However, a faint additional band was also noticed in the 9,11 , and 12 reactions equivalent in length to a 10 -mer (shown by the $\Delta$ arrow). This pattern was repeatedly observed in LASA reac- tions using PCR product but was not evident when using cloned DNA, suggesting that this band may be evidence of slight contamination. However, this by-product appears to cause minimal disturbance to the background readings (Figure 2).

\section{Overcoming Stem-Loop Formation with the Use of Competitive Oligonucleotides}

Various treatments were tried to remove any hairpin loop structures (data not shown), including the use of dimethyl sulfoxide (DMSO) $(0 \%-50 \%)$, formamide $(0 \%-50 \%), \mathrm{NaCl}(0-130$ $\mathrm{mM}), \mathrm{KCl}(0-130 \mathrm{mM}), \mathrm{MgCl}_{2}(0-110$ $\mathrm{mM}), \mathrm{MnCl}_{2}(0-22 \mathrm{mM})$, and S1 nuclease (0-2.5 U/reaction). Additionally, hybridization of the oligonucleotides to the template following the denaturation step was carried out over a slower period of 20, 40, and $60 \mathrm{~min}$. However, no significant improvement was observed with any of these treatments.

Successful reduction of background was achieved, however, using competitive oligonucleotides (Table 6). Competitive oligonucleotides were designed to span the upstream flanking and repetitive regions. These were designed to 


\section{Research Report}

Table 7. LASA Assay Results Performed on Bird Samples

\begin{tabular}{|c|c|c|c|c|c|c|c|c|c|c|c|}
\hline & \multicolumn{11}{|c|}{ Bird Sample } \\
\hline & 46766 & 48385 & 28102 & D782 & 46765 & 48937 & 48903 & D783 & MM100 & MM104 & 28103 \\
\hline Spacer 9 & 1.292 & 0.089 & 0.057 & 1.109 & 0.149 & 0.106 & 0.091 & 0.180 & 1.985 & 0.125 & 1.169 \\
\hline Spacer 10 & 1.763 & 1.113 & 1.293 & 0.200 & 1.319 & 1.460 & 1.099 & 1.965 & 3.264 & 1.146 & 1.401 \\
\hline Spacer 11 & 0.070 & 0.217 & 0.263 & 0.146 & 0.388 & 0.273 & 0.893 & 0.124 & 0.135 & 0.218 & 0.287 \\
\hline Spacer 12 & 0.061 & 0.103 & 0.142 & 0.096 & 0.146 & 0.111 & 0.100 & 0.381 & 0.273 & 0.083 & 0.366 \\
\hline LASA Genotype & 9,10 & 10,10 & 10,10 & 9,9 & 10,10 & 10,10 & 10,11 & 10,10 & 9,10 & 10,10 & 9,10 \\
\hline $\begin{array}{l}\text { Genotype } \\
\text { (6\% urea-PAGE gel }\end{array}$ & 9,10 & 10,10 & 10,10 & 9,9 & 10,10 & 10,10 & 10,11 & 10,10 & 9,10 & 10,10 & 9,10 \\
\hline
\end{tabular}

act as artificial templates to hybridize to any DNA sequences formed with hairpin loops by the template. We hypothesized that detection of the correct spacer addition would be favored, and any such loop formations would be present in low concentration. Absorbance readings of LASA reactions without competitive oligonucleotides showed background levels in the ATCC-9 well to be almost half of the correctly incorporated spacer oligonucleotide. Although the addition of competitive oligonucleotide slightly reduced the overall signal of the correct spacer addition, the background level for ATCC-9 was significantly decreased, providing much greater discrimination between correct and incorrect signal; in particular, $0.2 \mathrm{pmol}$ competitive oligonucleotide proved optimal, providing a reduction in the background signal by $85 \%$.

\section{Use of LASA to Determine \\ Microsatellite Length \\ Polymorphism in Birds}

Eleven bird samples were genotyped using both the conventional denaturing PAGE and the competitive LASA assay. Scoring of PAGE and LASA alleles were done by visual inspection in both cases.

The assay was performed on unknown samples comprised of both homozygous and heterozygous genotypes. The LASA absorbance readings were used to clearly distinguish between spacer lengths, with no significant confusion introduced by background levels (Table 7). Four heterozygous samples were apparent from the denaturing polyacrylamide gel. Samples 46766, 48903, and 28103 gave approximate 1:1 ratios between absorbance readings for the two allele lengths in the LASA method. Furthermore, sample MM100 gave signals of 2-fold difference. This corresponded exactly to the band intensities on the PAGE gel. All of the 11 samples resulted in the same genotype by both methods.

\section{DISCUSSION}

During the development and optimization of the LASA assay, several factors were shown to be critical for the successful measurement of microsatellite length. Although the period of denaturation and ligation were found to have no significant effect on the incorporation signal, an optimal ligation temperature of $65^{\circ} \mathrm{C}$ was required to maximize the ratio of absorbance readings from properly matched versus mismatched spacer oligonucleotides. It is anticipated that the ligation temperature will be oligonucleotide-specific and that it will need to be optimized for each new set of LASA oligonucleotides. A significant reduction in false incorporation signals was achieved by lowering the amount of template equivalent to $0.05 \mu \mathrm{L}$ PCR product (equivalent to approximately $250 \mathrm{fmol}$ ).

Although the initial assay used 99 rounds of alternate denaturation and ligation reactions, the investigation of the relationship between cycle number and absorbance signals was nonlinear (data not shown). A plateau was reached at approximately 20 cycles. We therefore selected 25 cycles to provide sufficient signal intensities (within 15$60 \mathrm{~min}$ of colorimetric detection) for clear positive results.

Although the LASA method consistently provided high signals for the correct incorporation of the spacer insert and, hence, the correct measurement of the number of tetramers within the repetitive sequence, template-dependent background signals for the incorrect spacer inserts were relatively high and unacceptable for accurate scoring of allele lengths. By using Southern blot analysis of the LASA products, it was shown that false incorporation of each particular spacer oligonucleotide in the separate reactions had occurred. This substantiated our theory that hairpin loops were being formed within either the template or the spacer oligonucleotide to accommodate the ligation of the three oligonucleotides. In addition, we were able to show that this phenomenon was also apparent when cloned DNA was used instead of PCR product, eliminating the possibility of contribution to absorbance signals by stutter artifacts produced during amplification of the PCR products. It was noted that these stem-loops were being formed to a greater extent in the template as opposed to the oligonucleotides, as demonstrated by the higher background level observed in the ATCC-9 reaction as compared to the ATCC-11 and ATCC-12 reactions. We attributed this to the formation of stem-loops within the repetitive region of the PCR prod- 
uct to accommodate the hybridization and subsequent ligation of the ATCC-9 spacer molecule to the capture and reporter flanking oligonucleotides.

Various treatments were attempted to eliminate the formation of these structures without success. A competitive ligation system was designed to absorb any templates containing hairpins, in particular targeting the formation of stemloops within the PCR product template. This was done by adding nonbiotinylated competitive oligonucleotides in the reaction, comprising the upstream flanking oligonucleotide (oligonucleotide "us") and the spacer oligonucleotide of larger and smaller size than the expected correct spacer insert. Since the competitive oligonucleotides were nonbiotinylated, there would be no interference with the capture of the true ligation event. This modification was successful in overcoming the background problem at a concentration of $0.2 \mathrm{pmol}$ each competitive oligonucleotide.

A precautionary note should be added in relation to the instability of the competitive oligonucleotides upon repeated thawing and freezing of stock solutions. We have previously observed the reappearance of background signals due to this occurrence; therefore, we stress the need for the inclusion of a positive control to monitor this effect.

Thus, three critical factors were identified that contributed to the decrease in background signals in the LASA assay and were overcome by: $(i)$ the optimization of ligation reaction, in particular, the ligation temperature and number of cycles of denaturation-ligation; (ii) the addition of competitive oligonucleotide to absorb any stemloops formed within the template or spacer molecule; and (iii) the reduction of the amount of template available for the LASA reaction.

The LASA procedure was used on a set of 11 bird samples to validate its performance as a genotyping method. Allele sizes were also determined by conventional denaturing gel electrophoresis. In comparing both methods, all DNA samples matched genotypes perfectly. Sample 28103 demonstrated absorbance signals of approximately equal values as expected for a heterozygous signal. However, sample MM100 produced signals of 2-fold increase for one allele as compared to the other. The corresponding sample on the denaturing gel confirmed the presence two bands, one of which was stronger in intensity than the other.

Two recent publications have utilized a ligase-based technology to detect both mononucleotide and dinucleotide microsatellite repeats. Zirvi and co-workers $(25,26)$ used a PCR/ligase detection reaction (PCR/LDR), comprised of a fluorescently labeled upstream (discriminating) oligonucleotide of varying lengths and a phosphorylated downstream (common) oligonucleotide. Results demonstrated that the majority of the ligated product was of the correct size; however, incorrect ligations were produced, with levels being as high as $25 \%$ of the ligated product of correct size. Our data substantiate Zirvi and co-workers' postulate that misligation errors were due to the formation of stem-loops. They were able to reduce the background to less than $15 \%$ by using modified nucleosides, in particular the nitroimidazole nucleoside analog to increase fidelity of ligation. Other improvements included the addition of formamide in the ligation buffer, as well as increasing the ligation temperature. Although we found no significant effect with the addition of formamide, we demonstrated that the ligation temperature was a critical factor for the LASA reaction and would need to be considered for each new set of LASA oligonucleotides.

The LASA procedure is neither laborious nor time-consuming (maximum 3 h), particularly when one compares it to current traditional gel-based microsatellite detection methods. In addition to its simplicity and ease of setup, the LASA methodology is highly desirable as an assay for routine laboratories, without the necessity for dedicated and expensive instrumentation, with the exception of a colorimetric plate reader.

This method has been devised to utilize known and characterized microsatellite markers. There will be instances when this methodology will not be an acceptable tool, such as for compound or interrupted microsatellites, or extremely long microsatellite regions, or markers with a large number of alleles.

The LASA assay has several significant advantages over other methods that 


\section{Research Report}

are currently used for genotyping of microsatellite length polymorphisms, such as $(i)$ it circumvents the need for electrophoresis, a labor-intensive and timeconsuming process; ( $i i)$ it utilizes a fluorescently labeled reporter molecule, allowing for direct fluorescence detection rather than colorimetric detection as used here; and (iii) being an ELISAbased assay, it is readily adaptable to automation. When applied to allelic discrimination, this technology is ideal for high throughput as required in largescale genotyping screening. The use of a 96-well plate format further enhances its advantage as a large-scale screening tool. There is scope for its use with alternative solid-phase media, such as oligonucleotide array-based technology, because streptavidin-coated microplates are limiting in their binding capacities. Its applicability to medical diagnostics is highly evident. Further applications of this method may include its use in the forensics field for personal identification. Furthermore, novel trinucleotide repeat expansions have been associated with a number of disorders such as Huntington's disease and Fragile X syndrome. Current diagnosis also relies upon the fractionation of PCR-amplified alleles by denaturing gel electrophoresis and Southern blotting. By eliminating the use of electrophoresis, the LASA methodology reduces the amount of technical labor and time for analysis.

\section{ACKNOWLEDGMENTS}

We are grateful for the Major Mitchell Cockatoo samples kindly donated by Dick Schodde from the Australian National Museum and Julie Macaranas from Queensland University of Technology in association with the Queensland Department of Environment and Heritage. David Growth from the University of Western Australia graciously provided us with the MM211 microsatellite sequence.

\section{REFERENCES}

1.Amos, W., S.J. Swacer, R.W. Feakes, and D.C. Rubinsztein. 1993. Microsatellites show mutational bias and heterozygote instability. Nat. Genet. 13:390-391.

2.Campuzano, V., L. Muntermini, M.D. Mot- to, L. Pianese, M. Cossee, F. Cavalcanti, E. Monros, F. Rodius, F. Duclos, and A. Monticelli. 1996. Friedrich's ataxia: autosomal recessive disease caused by an intronic GAA triplet repeat expansion. Science 271:14231427.

3.Gill, P., A.J. Jeffreys, and D.J. Werrett. 1985. Forensic applications of DNA 'fingerprints'. Nature 318:577-579.

4.Hearne, C.M., S. Ghosh, and J.A. Todd. 1992. Microsatellites for linkage analysis of genetic traits. TIG 8:288-294.

5.Huntington's Disease Collaboration Research Group. 1993. A novel gene containing a trinucleotide repeat that is expanded and unstable on Huntington disease chromosomes. Cell 72:971-983.

6.Jeffreys, A.J., V. Wilson, R. Neuman, and J. Keyte. 1989. Amplification of human minisatellites by the polymerase chain reaction: towards DNA fingerprinting of single cells. Nucleic Acids Res. 16:10953-10971.

7.Klinkicht, M. and D. Tautz. 1992. Detection of simple sequence length polymorphism by silver staining. Mol. Ecol. 1:133-134.

8.Knowles, J.A., V.J. Vicland, and T.C. Gilliam. 1992. Perils of gene mapping with microsatellite markers. Am. J. Hum. Genet. 51:905-909.

9.Kristensen, V.N. and A.B. Dale. 1997. Improved electrophoretic separation of polymorphic short tandem repeats in agarose gels using bis-benzimide. Biotechnology 23:634636.

10.Lins, A.M., C.J. Sprechen, C. Puers, and J.W. Schumm. 1996. Multiplex sets for the amplification of polymorphic short tandem repeat loci-silver stain and fluorescence detection. Biotechnology 20:882-888.

11.Longmire, J.L., M. Maltbie, R.W. Pavelka, L.M. Smith, S.M. Witte, O.A. Ryder, D.L. Ellsworth, and R.J. Baker. 1993. Gender identification in birds using microsatellite DNA fingerprint analysis. Auk 110:378-381.

12.MacDonald, D.B. and W.K. Potts. 1994. Cooperative display and relatedness among males in a lek-mating bird. Science 266:10301032.

13.Mathies, R.A. 1995. Biomolecular vibrational spectroscopy. Methods Enzymol. 246:377-389.

14.Primmer, C.R., A.P. Moller, and H. Ellegren. 1995. Resolving genetic relationships with microsatellite markers: a parentage testing system for the swallow Hirundo rustica. Mol. Ecol. 4:493-498.

15.Rousseau, F., D. Heitz, I. Oberle, and J.L. Mandel. 1991. Selection in blood cells from female carriers of the fragile $\mathrm{X}$ syndrome: inverse correlation between age and proportion of active $\mathrm{X}$ chromosomes carrying the full mutation. J. Med. Genet. 28:830-836.

16.Roy, M.S, E. Geffen, D. Smith, E.A. Ostrander, and R.T.K. Wayne. 1994. Patterns of differentiation and hybridisation in North American wolf-like canids, revealed by analyses of microsatellite loci. Mol. Biol. Evol. 11:553-570

17.Strassman, J.E., C.R. Solis, J.M. Peters, and D.C. Queller. 1996. Strategies for finding and using highly polymorphic DNA microsatellite loci for studies of genetic related- ness and pedigrees. In J.D. Ferrarris and S.R. Palumbi (Eds.), Molecular Zoology: Advances, Strategies and Protocols. Wiley-Liss, New York.

18.Sutherland, G.R, J.C. Mulley, and R.I. Richards. 1993. Fragile X syndrome: the most common cause of familial intellectual handicap. Med. J. Aust. 158:482-485.

19.Taranenko, N.I., N.T. Potter, S.L. Allman, V.V. Golouleu, and C.H. Chen. 1999. Detection of trinucleotide expansion in neurodegenerative disease by matrix-assisted laser desorption/ionisation time of flight mass spectroscopy. Genet. Anal. Biomol. Eng. $14: 25-31$

20.Tautz, R. 1989. Hypervariability of simple sequences as a general source for polymorphic DNA markers. Nucleic Acids Res. 17:64636471

21.Taylor, A.C., W.B. Sherwin, and R.K. Wayne. 1994. Genetic variation of microsatellite loci in a bottlenecked species: the northern hairy-nosed wombat Lasiorhinus kreffti. Mol. Ecol. 3:277-290.

22.Tegelstrom, H. 1986. Mitochondrial DNA in natural populations: an improved routine for screening of genetic variation based on sensitive silver staining. Electrophoresis 7:226229.

23.Tereba, A., K.A. Micka, and J.W. Schumm. 1998. Re-use of denaturing polyacrylamide gels for STR analysis. Biotechnology 25:892897.

24.Vuillaume, I., S. Schraen, J. Rousseaux, and B. Sablonniere. 1998. Simple non-isotopic assays dor detection of $(\mathrm{CAG})_{\mathrm{n}}$ repeat expansions associated with seven neurodegenative disorders. Diagn. Mol. Pathol. 7:174179.

25.Zirvi, M., T. Nakayama, T. McCaffrey, P. Paty, and F. Barany. 1999a. Ligase-based detection of mononucleotide repeat sequences. Nucleic Acids Res. 27:e40.

26.Zirvi, M., D.E. Bergstrom, A.S. Saurage, R.P. Hammer, and F. Barany. 1999b. Improved fidelity of thermostable ligases for detection of microsatellite repeat sequences using nucleoside analogs. Nucleic Acids Res. 27:e41

Received 5 July 2000; accepted 23 January 2001.

Address correspondence to:

Dr. Veronica Brockhurst

CRC for Diagnostic Technologies

School of Life Sciences

Queensland University of Technology

GPO Box 2434

Brisbane. Qld. 4001, Australia

e-mail:v.futo@qut.edu.au 